\title{
CO-JP-4
}

\section{Simultaneous Optimization of Variable Blank Holder Force Trajectory and Tools Motion in Deep Drawing via Sequential Approximate Optimization*}

\author{
Jirasak SRIRAT ${ }^{* *}$, Satoshi KITAYAMA*** and Koetsu YAMAZAKI*** \\ "Graduate School of Natural Science and Technology, Kanazawa University, Kakuma-machi, Kanazawa, 920-1192 Japan \\ E-mail: jirasak@stu.kanazawa-u.ac.jp \\ -. Kanazawa University, Kakuma-machi, Kanazawa, 920-1192, Japan
}

\begin{abstract}
In this paper, variable blank holder force (VBHF) trajectory and tools motion are optimized simultaneous by a sequential approximate optimization (SAO) with radial basis function (RBF) network. In deep drawing, wrinkling and tearing are major defects that are strongly avoided in sheet forming. These defects are then taken as the objectives directly. The Forming Limit Diagram (FLD) is employed to evaluate the risk of wrinkling and tearing quantitatively. The design variables are taken so as to optimize the VBHF trajectory and the tools motion simultaneously. In numerical examples, a square cup deep drawing is handled. The optimum result shows that simultaneous optimization of the VBHF trajectory and the tools motion result in the successful deep drawing. In particular, the forming energy can be drastically reduced, in comparison with only the optimization of VBHF trajectory. This result implies that the simultaneous optimization of both VBHF trajectory and tools motion will be effective approach to reduce the forming energy.
\end{abstract}

Key words: Deep Drawing, Computer Aided Engineering, Engineering Optimization, Variable Blank Holder Force, Tools Motion

\section{Introduction}

Wrinkling and tearing are major defects in sheet metal forming. In order to avoid these defects before manufacturing, numerical simulation based on Finite Element Analysis (FEA) is a powerful tool ${ }^{(1)}$. There are many factors affecting the wrinkling and the tearing, such as tools geometry, blank holder force (BHF), lubrication, and so on. Among them, BHF is one of the effective factors ${ }^{(2)}$. Large BHF leads to the tearing, while small BHF will result in the wrinkling. An appropriate BHF without these defects can be found through numerical simulation, but the numerical simulation is an expensive and time-consuming task.

Variable BHF (VBHF) approach that BHF varies through the punch stroke is an attractive technique for avoiding the tearing and the wrinkling, and many approaches have been proposed ${ }^{(3)-(13)}$. These approaches can be roughly classified into two categories: One is to construct the closed-loop type algorithm for $\mathrm{VBHF}^{(3)-(6)}$, and the other is based on the response surface approach $^{(7)(13)}$. The characteristics of two approaches are well summarized in Refs. $(6,13)$, then we do not described them here in detailed. When the closed-loop type algorithm is employed, the state of blank should be always checked during the forming process. As the result, many numerical simulation runs are required to obtain VBHF with the closed-loop type algorithm. In addition, an initial BHF plays an important role in the VBHF trajectory, but no clear guide determining the initial BHF is described. Thus, the initial BHF should be determined by trial-and-error method. On the other hand, the response surface method (RSM) can drastically reduce the number of numerical simulation runs, in comparison with the closed-loop type algorithm. In recent, a sequential approximate optimization (SAO) approach has gained its popularity. It has been reported that SAO is attractive approach in sheet forming simulation ${ }^{(14)}$. In SAO, a small-size design of experiment (DOE) is first employed to construct the response surface. The optimum of the response surface can then be found. If the 
terminal criteria determined by the designer are satisfied, the SAO algorithm will be terminated. Otherwise, a few new sampling points are added to improve the accuracy. Through the above iterative process, a highly accurate global optimum can be found. We have developed the SAO system with the radial basis function (RBF) network ${ }^{(15)}$, and have applied to the optimization of VBHF trajectory in deep drawing ${ }^{(13)}$.

In the VBHF trajectory problem, a pre-determined tools motion is employed. Thus, BHF varies through the punch stroke, while the tools motion is determined in advance. If the blank holder motion is also controlled as well as the BHF through the punch stroke, it is expected that the product quality will be improved. Nowadays, energy consumption is a major concern. If the simultaneous control of both the BHF and the tools motion can reduce the forming energy, one of the energy consumption issues will be resolved. In this paper, we aim to control and optimize the BHF and the tools motion simultaneousty. There are few papers focusing on such controlling technique. In Refs. $(16,17)$, in order to eliminate wrinkling during deep drawing, the BHF and the punch speed are controlled separately. The closed-loop type algorithm for wrinkling elimination called the alternating blank holder control method has been developed. The illustrative example taken from Ref. (16) is shown in Fig.1, in which "SPD" denotes the punch speed. Also, "Unload" denotes the small BHF.

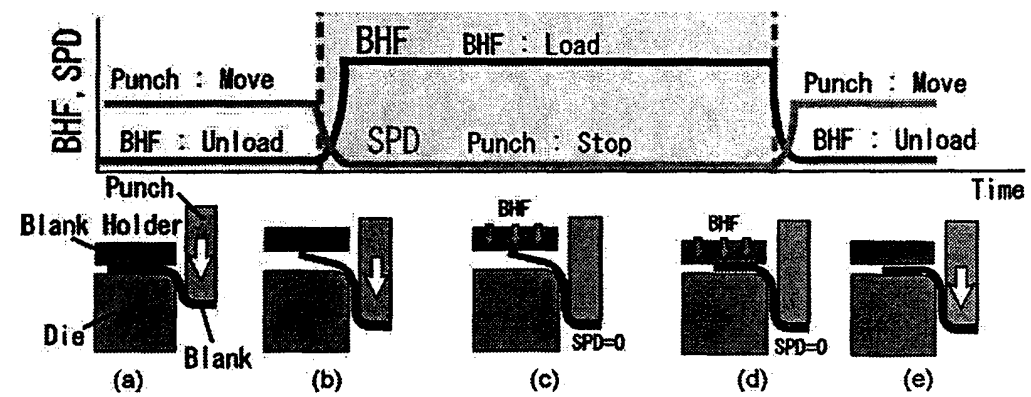

(a)

Fig.1 Alternating blank holder control method ${ }^{(16)}$

The algorithm starts with an extremely low BHF. The punch also moves as shown in Fig. 1(a). When the wrinkling can be observed (Fig. 1(b)), the punch is stopped and the large BHF is applied (Fig. 1(c)). The wrinkling is then eliminated with large BHF (Fig.1(d)), and low BHF is applied and the punch moves again (Fig. 1(e)).

Unlike Ref. (2)-(13), this method controls both the BHF and the punch speed, and is so attractive. Unfortunately, following issues are unresolved:

P1: This algorithm starts with an extremely low BHF. As described above, initial BHF plays an important role, and is difficult to determine in advance.

P2: The BHF and the punch speed are controlled separately, as shown in Fig.1. Thus, this method does not control them simultaneously. It is possible to control and optimize them simultaneously. In order to reduce the number of numerical simulation runs, the response surface approach is preferable.

This paper considers the simultaneous optimization of both VBHF trajectory and the tools motion via the SAO with the RBF network. In this paper, the tearing and the wrinkling are considered as the objective functions, unlike Ref. (13). The forming limit diagram (FLD) is employed to evaluate these objectives quantitatively ${ }^{(18)}$.Thus, multi-objective design optimization is formulated. The design variables are (1) BHFs at each stroke-step, (2) the velocity of tools motion, and (3) the stroke length to switch the BHFs and the tools motion. The weighted $l p$ norm method is employed to find the optimal solution. By employing the weighted $l p$ norm method, the SAO system for a single design optimization can be easily extended to the multi-objective design optimization.

The rest of paper is organized as follows: In section 2, the FEA model is briefly described. The multi-objective design optimization is also formulated. In section 3, the SAO procedure is concretely described. The validity is examined through numerical simulation section 4. LS-DYNA, which is one of the dynamic explicit FEA code is employed in the numerical simulation.

\section{Finite Element Analysis Model and Multi-objective Design Optimization}

\subsection{Finite Element Analysis Model}

The FEA model taken from Ref. $(6,13)$ is shown in Fig. 2. The material properties are listed in Table 1, which are obtained through the experiment. We have already examined the validity of the FEA model through an experiment with a constant blank holder force (BHF) $(100[\mathrm{kN}])^{(6)}$. SPFC440 (Steel Plate Formability Cold) was selected as the test material. 
The blank size is $185[\mathrm{~mm}] \times 185[\mathrm{~mm}]$, and the initial thickness is $1.20[\mathrm{~mm}]$. In numerical simulation, $1 / 4$ model is employed for the geometrical symmetry. Thus, a blank size of $92.5[\mathrm{~mm}] \times 92.5[\mathrm{~mm}]$ is employed. The finite element models for the tools and the blank used in the square cup deep drawing are also shown in Fig. 2. The element type and the number of finite elements are tabulated in Table 2. A constant friction coefficient $\mu$ of 0.1 is used for all contact surfaces: blank/blank holder, blank/punch, blank/die, and blank/counter punch. A Belytschko-Tsay shell element with seven integration points across the thickness is used for the shell mesh of the blank. The relationship of stress-strain is approximately obtained from database in the LS- DYNA as follows:

$$
\sigma=793 \varepsilon^{0.189}
$$
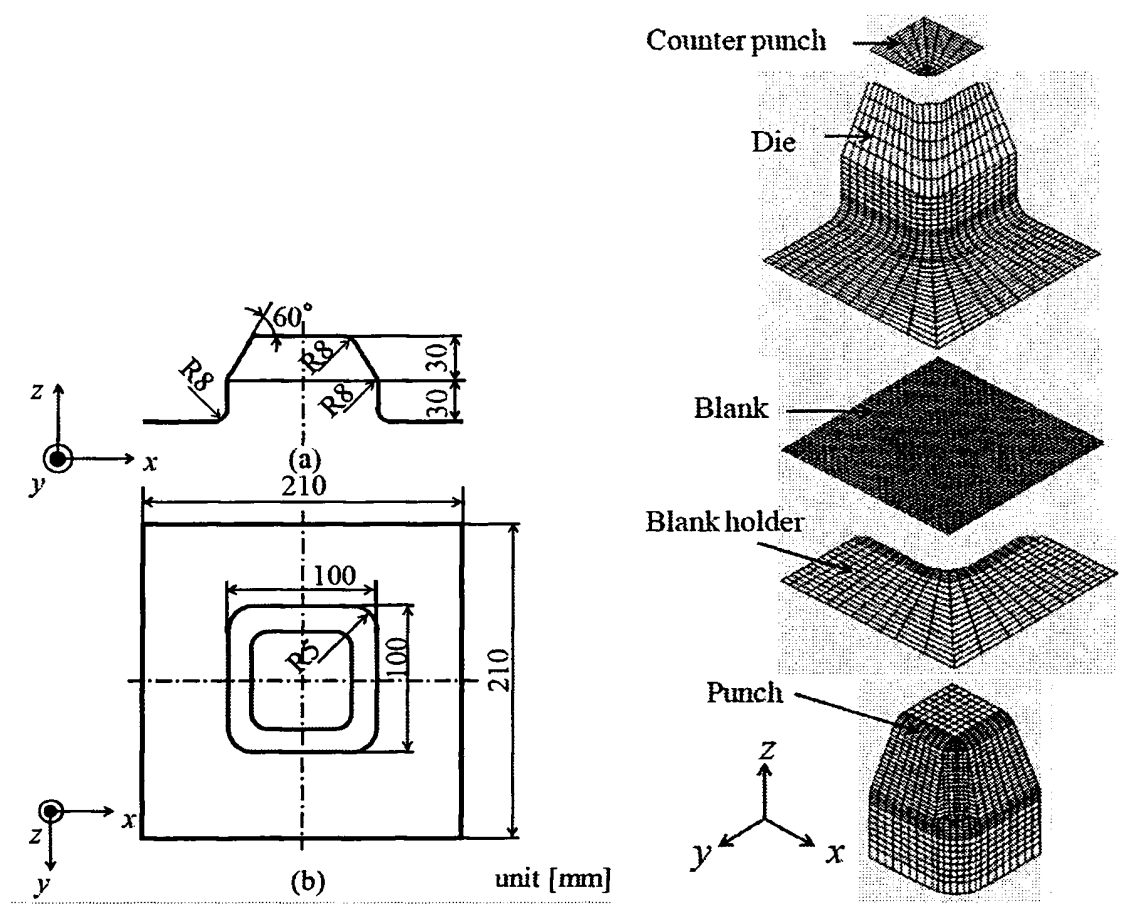

Fig. 2 Finite element model for square cup deep drawing process

The punch is fixed and the BHF is applied in the positive $z$-direction. The counter punch, the die, and the blank holder drop to the negative $z$-direction. At the bottom dead center, the counter punch, the die, and the blank holder move upwards.

Table 1 Mechanical properties of blank sheet (SPFC 440)

\begin{tabular}{|c|c|}
\hline Density: $\rho\left[\mathrm{kg} / \mathrm{mm}^{3}\right]$ & $7.84 \times 10^{-6}$ \\
\hline Young's modulus: $E[\mathrm{MPa}]$ & $2.06 \times 10^{5}$ \\
\hline Poisson's ratio: $\nu$ & 0.3 \\
\hline Yield stress: $\sigma_{Y}[\mathrm{MPa}]$ & 353 \\
\hline Tensile strength: $\sigma_{T}[\mathrm{MPa}]$ & 479 \\
\hline Normal anisotropy coefficient: $r$ & 0.98 \\
\hline Strain hardening coefficient: $n$ & 0.189 \\
\hline
\end{tabular}

Table 2 Element type for numerical simulation

\begin{tabular}{|l|c|c|}
\hline & Element type & Number of finite elements \\
\hline Counter punch & Rigid & 120 \\
\hline Dies & Rigid & 924 \\
\hline Blank & Shell (Belytschko-Tsay) & 2116 \\
\hline Blank holder & Rigid & 432 \\
\hline Punch & Rigid & 962 \\
\hline
\end{tabular}




\subsection{Multi-objective Design Optimization}

As described in introduction, two objective functions that are the wrinkling and the tearing are considered. Also, the design variables are taken so as to optimize the VBHF trajectory and tools motion simultaneously.

\subsubsection{Objective functions}

In order to evaluate the risk of wrinkling and tearing quantitatively, the FLD is employed. In the FLD the strain states of all elements are plotted into major-minor strain plane. Let us consider the FLD as shown in Fig. 3.

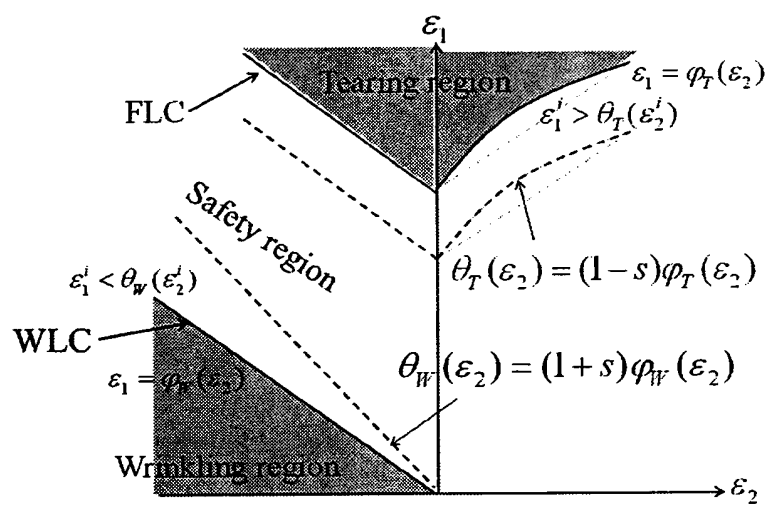

Fig. 3 Forming Limit Diagram (FLD)

The stains in formed element are analyzed and compared against the Forming Limit Curve (FLC, as shown in Fig. 3). Then, the following FLC in the principal plane of logarithmic strains was proposed by Hillman and Kubli ${ }^{(19)}$.

$$
\varepsilon_{1}=\varphi_{T}\left(\varepsilon_{2}\right), \varepsilon_{1}=\varphi_{W}\left(\varepsilon_{2}\right)
$$

where $\varphi_{T}$ is one FLC which controls the tearing, and $\varphi_{W}$ is the Wrinkling Limit Curve (WLC) which controls the wrinkling. Both of them depend on the material properties, which are generally given as knots data in tables. Then, the following safety FLC is defined:

$$
\begin{aligned}
& \theta_{T}\left(\varepsilon_{2}\right)=(1-s) \varphi_{T}\left(\varepsilon_{2}\right) \\
& \theta_{W}\left(\varepsilon_{2}\right)=(1+s) \varphi_{W}\left(\varepsilon_{2}\right)
\end{aligned}
$$

where $s$ represents the safety tolerance, and is defined by the engineers. In this paper, $s$ is set to 0.2 . If some strain state of an element lies above the safety FLC $\theta_{T}$, it is expected that a risk of tearing can be observed. Similarly, a risk of wrinkling can be assumed if the strain state of an element lies in the wrinkling region below the safety $\operatorname{WLC}\left(\theta_{W}\right)$.

In order to clarify both risks, we examine separately the risk of both wrinkling and tearing. Referring to Ref. (20), the following two objective functions are formulated:

\section{For wrinkling:}

$$
f_{1}(x)=\left\{\begin{array}{cc}
\sum_{i=1}^{n e l m}\left[\varepsilon_{1}^{i}-\theta_{W}\left(\varepsilon_{2}^{i}\right)\right] \exp \left[\varepsilon_{1}^{i}-\theta_{W}\left(\varepsilon_{2}^{i}\right)\right] & \varepsilon_{1}^{i}<\theta_{W}\left(\varepsilon_{2}^{i}\right) \\
0 & \text { Otherwise }
\end{array}\right.
$$

\section{For tearing:}

$$
f_{2}(x)=\left\{\begin{array}{cc}
\sum_{i=1}^{n e l m}\left[\varepsilon_{1}^{i}-\theta_{T}\left(\varepsilon_{2}^{i}\right)\right] \exp \left[\varepsilon_{1}^{i}-\theta_{T}\left(\varepsilon_{2}^{i}\right)\right] & \varepsilon_{1}^{i}>\theta_{T}\left(\varepsilon_{2}^{i}\right) \\
0 & \text { Otherwise }
\end{array}\right.
$$

where nelm denotes the total number of finite elements of the blank. 


\subsubsection{Design variables}

The design variables are taken so as to optimize the VBHF trajectory and tools motion simultaneously. Let us explain how to take the design variable using Fig. 4(a) and (b).
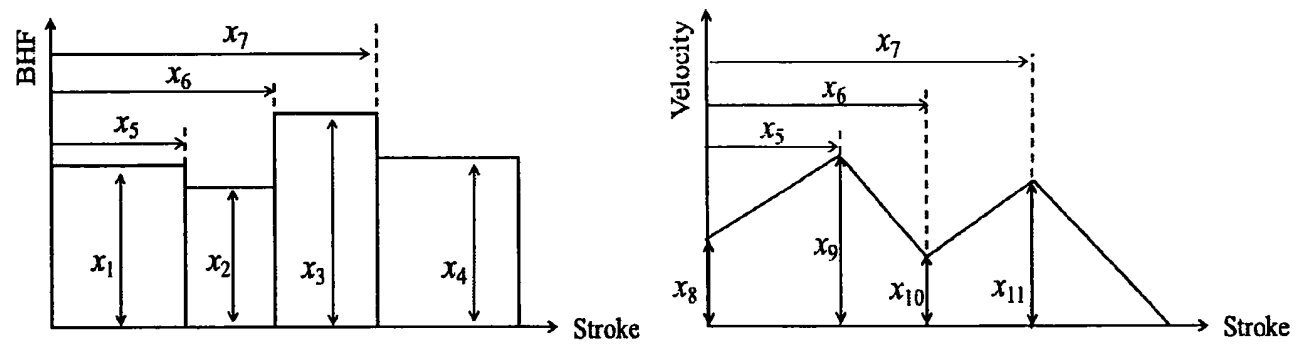

(a) VBHF trajectory

(b) Tools motion

Fig. 4 Design variables

The total stroke is divided into 4 sub-strokes for VBHF trajectory as shown in Fig. 4(a), and each BHFs at sub-stroke is taken as the design variables. Also, each sub-stroke length is taken as the design variables.

Next, the tools motion is considered. In this paper, the motion of the counter punch, the die, and the blank holder shown in Fig. 2 is called the tools motion. In the tools motion, as shown in Fig. 4(b), initial velocity at each sub-stroke is taken as the design variables. Note that the design variable $x_{5}, x_{6}$, and $x_{7}$ are commonly used. As the result, the BHF and the tools motion are controlled and optimized simultaneously.

\subsubsection{Formulation as multi-objective design optimization}

The simultaneous optimization of both the VBHF trajectory and the tools motion is formulated as follow:

$$
\begin{aligned}
& \left(f_{2}(x), f_{2}(x)\right) \rightarrow \min \\
& x_{i}^{L} \leq x_{i} \leq x_{i}^{U} \quad i=1,2, \cdots, 11
\end{aligned}
$$

where $x_{i}$ is the $i$-th design variable. $x_{i}^{L}$ and $x_{i}^{U}$ represent the lower and upper bounds of the $i$-th design variables, respectively. It is clear from Eqs. 7 and 8 that above design optimization problem has many solutions called the Pareto-optimal solution. There are many methods to find the Pareto-optimal solution such as the Evolutionary Algorithms, the Interactive method, and the scalarization method. In this paper, the weighted $l p$ norm method is used to find the Pareto-optimal solution ${ }^{(21)}$.

\subsubsection{The weighted $l p$ norm method}

The weighted $l p$ norm method is one of the scalarization methods in multi-objective design optimization. In this method, the following augmented objective function $F(x)$ is constructed to find the Pareto-optimal solution:

$$
\|\boldsymbol{F}(x)\|_{p}^{w}=\left[\sum_{i=1}^{K}\left(w_{i} f_{i}(x)\right)^{p}\right]^{1 / p} \rightarrow \min
$$

where $K$ denotes the number of objective functions, and $p(\geq 1)$ is the parameter. $w_{i}$ denotes the weight of the $i$-th objective function. Therefore, multi-objective design optimization problem is transformed into a single objective optimization problem. Also, unlike the weighted sum, this method can support the non-convex Pareto frontier with $p \geq 2$, as shown in Fig. 5. In this method, the weight $w_{i}$ and the parameter $p$ should be adjusted in advance. For simplicity, the same weight $\left(w_{1}=w_{2}=0.5\right)$ are assigned, and $p$ is set to 2 . 


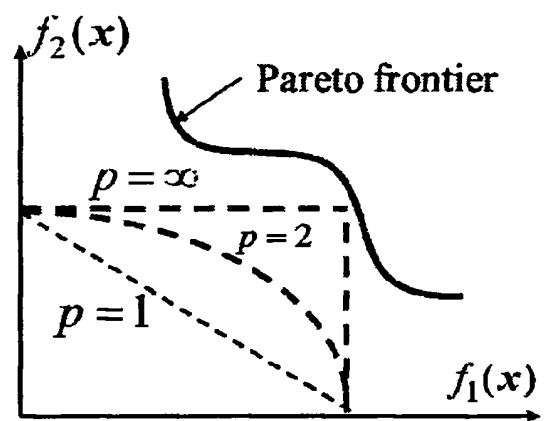

Fig. 5 Support of the pareto-frontier by the weighted $l p$ norm method

\section{Sequential Approximate Optimization with Radial Basis Function network}

The multi-objective design optimization can be transformed into the single objective optimization problem. Then, the SAO with the RBF network developed by authors can be used. The detailed procedure is summarized as follows:

STEP1: Initial sampling points are generated by the latin hypercube design (LHD).

STEP2: Two objective functions are evaluated by the FLD, and are approximated by the RBF network.

STEP3: Augmented objective function is constructed by the weighted $l p$ norm method. The augmented objective function is minimized.

STEP4: The optimum of the augmented objective function added as the new sampling point for improving the local accuracy.

STEP5: In order to find the unexplored region, the density function is constructed. The optimum of the density function is added as the new sampling point for improving the global approximation.

STEP6: If terminal criterion is satisfied, the algorithm will be terminated. Otherwise we return to STEP2.

\section{Numerical Results}

\subsection{VBHF trajectory and tools motion by the proposed approach}

20 initial sampling points are generated by the LHD. The number of maximum sampling points that is equivalent to the number of function evaluations is used as the terminal criterion of SAO algorithm, and is set to 130 . The side constraints of design variables are set as follows:

$$
\begin{aligned}
& 20 \leq x_{1}, x_{2}, x_{3}, x_{4} \leq 150 \quad \text { (For BHF) } \\
& 0 \leq x_{5} \leq 20,20 \leq x_{6} \leq 40,40 \leq x_{7} \leq 62 \quad \text { (For sub-stroke length) } \\
& 0 \leq x_{8}, x_{9}, x_{10}, x_{11} \leq 350 \quad \text { (For tools motion velocity) }
\end{aligned}
$$

The VBHF trajectory and the tools motion at the optimum are shown in Fig. 6, in which the solid and the dashed line represent the VBHF trajectory and the tools motion, respectively.

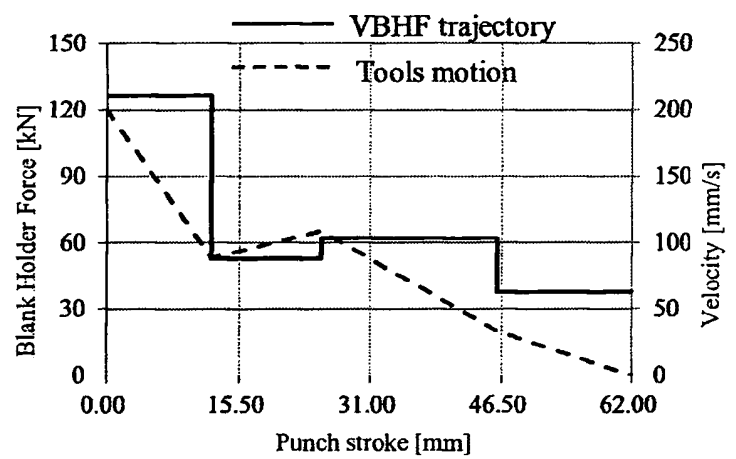

Fig. 6 VBHF trajectory and the tools motion at the optimum solution

It is clear from Fig. 6 that the high initial BHF is applied with the high velocity of tools motion at the beginning. Then, as 
the punch stroke goes on, the BHF is gradually decreased so as to stimulate the material flow into a die cavity.

Next, the following two criteria are used to check the wrinkling and the tearing: One is the use of FLD, and the other is the minimum thickness for tearing and the geometrical distance for wrinkling. In particular, the wrinkling can be also measured with the distance between the die and the blank holder. Based on Refs. $(6,13)$, the critical values for the tearing and the wrinkling are set to $0.9[\mathrm{~mm}]\left(=t_{o} \times 0.75\right)$ and $1.44[\mathrm{~mm}]\left(=t_{o} \times 1.2\right)$, respectively. Figure 7 (a) and (b) shows the FLD and the thickness distribution, respectively. It is clear from Fig. 7 that no tearing and wrinkling can be observed. It is found from these figures that at least the proposed approach can form without major defects.

Finally, the accuracy of the response surface of both objectives is examined. Figure 8 shows the accuracy of the response surfaces, in which the left and right hand side represent the wrinkling and the tearing, respectively. It is clear from Fig. 8 that highly accurate response surfaces have been obtained.

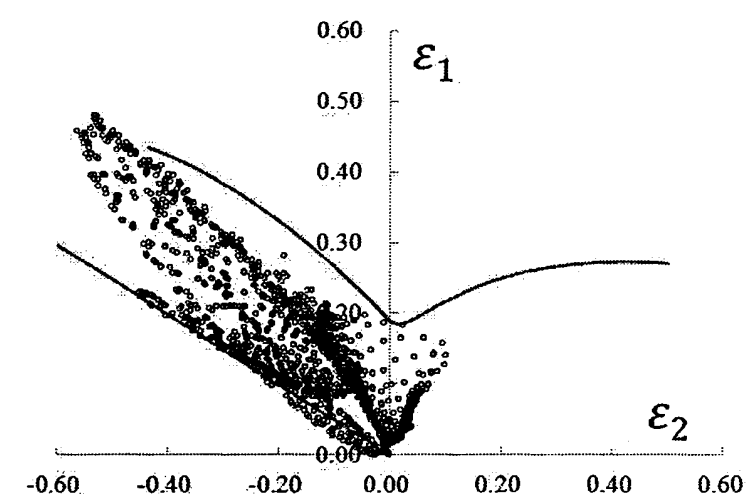

(a) FLD at the optimum solution

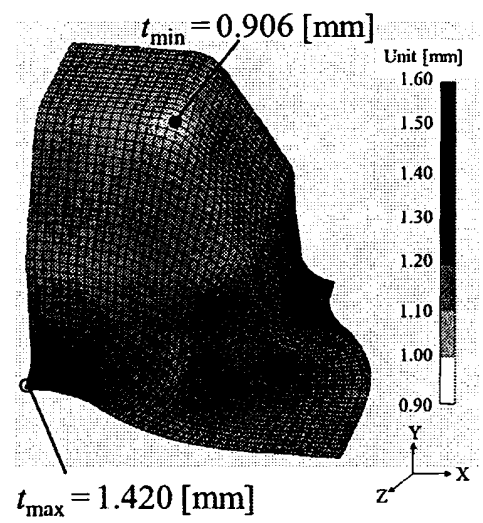

(b) Thickness distribution

Fig. 7 FLD and thickness distribution at the optimum solution

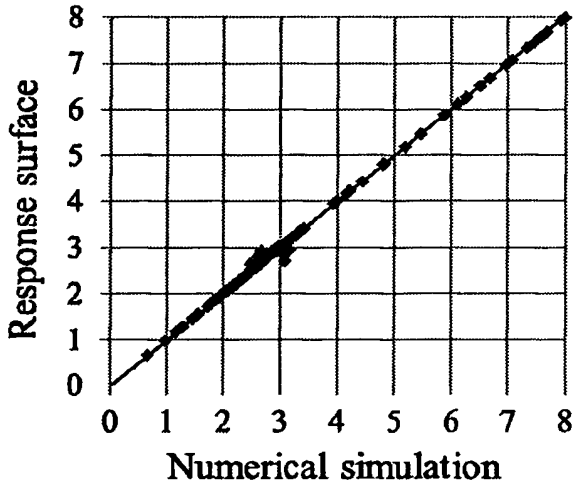

(a) Wrinkling

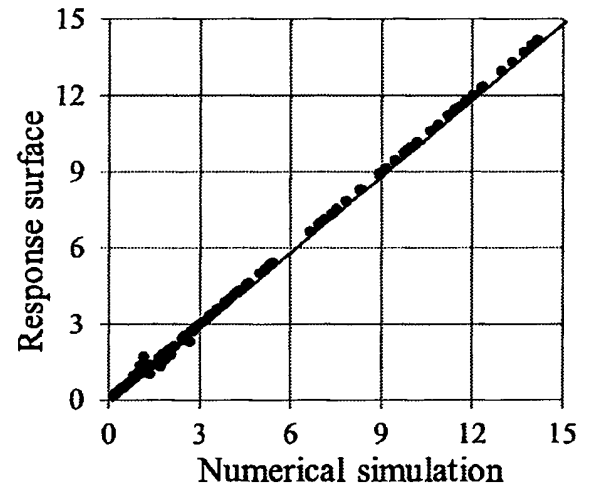

(b) Tearing

Fig. 8 Accuracy of the response surfaces

\subsection{Comparison between proposed approach and previous work}

We have applied the proposed SAO with the RBF network to the optimization of the VBHF trajectory ${ }^{(13)}$, in which the same FEA model was used. On the other hand, the tools motion was pre-determined. In Ref. (13), the objective function is to minimize the thickness deviation under the tearing and wrinkling constraints. Since the design optimization problem is completely different, it may meaningless to compare the VBHF trajectories of both approaches. However, some new ideas for resolving the energy consumption issues could be obtained.

The VBHF trajectories of both approaches are shown in Fig. 9, in which the solid and the dashed line represent the VBHF trajectory of the proposed approach and Ref. (13), respectively. In addition, Fig. 10 shows the tools motion.

It can be found from Fig.9 that both trajectories are completely different. In particular as the punch stroke goes on, the BHF is gradually decreased in the proposed approach, while the higher BHFs are applied in Ref. (13). The tools motion is also different. In Ref. (13), the velocity of tools motion is gradually decreased, while the velocity is increased at a certain sub-stroke in the proposed approach. 
In Fig. 9, the gray part is considered as the forming energy. Then, the forming energy is calculated, and is listed in Table 3. It is clear from Table 3 that the proposed approach can drastically reduce the forming energy, in comparison with the one of Ref. (13). Therefore, $31 \%$ forming energy reduction can be achieved. This result implies that the simultaneous optimization of VBHF trajectory and the tools motion can reduce the forming enegy, compared with only the optimization of VBHF trajectory.

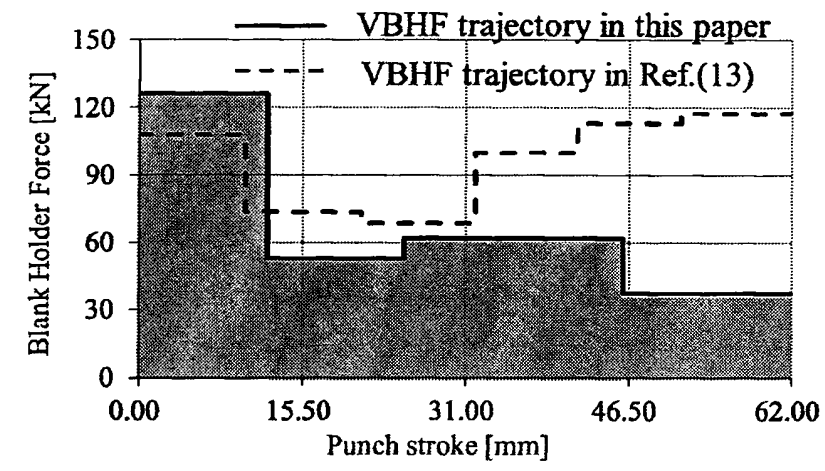

Fig. 9 VBHF trajectories

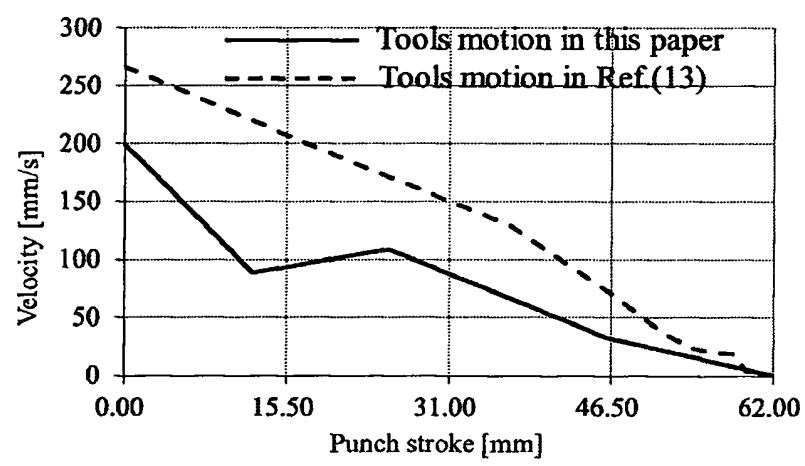

Fig. 10 Tools motion

Table 3 Comparison of forming energy

\begin{tabular}{c|c}
\hline & Forming Energy $[\mathrm{kN} \cdot \mathrm{mm}]$ \\
\hline Previous approach (Ref. 13) & 5,969 \\
\hline Proposed approach & 4,117 \\
\hline
\end{tabular}

\section{Conclusions}

In this study, the simultaneous optimization of the VBHF trajectory and the tools motion in deep drawing was performed by the SAO with the RBF network. The wrinkling and tearing are directly considered as the objective functions. The total stroke divided into some sub-strokes, and the BHF at each sub-stroke and sub-stroke length were taken as the design variables. Also, in order to control the tools motion, the velocity at each sub-stroke was taken as the design variables. Then, the multi-objective design optimization was formulated. In order to apply the SAO for a single objective optimization problem, the weighted $l p$ norm method was employed.

The VBHF trajectory and the tools motion were compared with the previous work by the authors. It can be from the comparison that the proposed approach could be drastically reduced the forming energy. This result suggested that the simultaneous optimization of VBHF trajectory and the tools motion can be more effective approach, compared with only the optimization of the VBHF trajectory.

\section{References}

1. Gantar, G., Pepelnjak, T., and Kuzman, K., Optimization of Sheet Metal Forming Process by the use of Numerical Simulations, 
Journal of Materials Processing Technology, Vol. 130-131, (2002), pp. 54-59.

2. Obermeyer, E.J., and Majlessi, S.A., A Review of Recent Advances in the Application of Blank-Holder Force towards Improving the Forming Limits of Sheet Metal Parts, Journal of Materials Processing Technology, Vol. 75, No. 1-3, (1998), pp. 222-234.

3. Sheng, Z.Q., Jirathearanat, S., and Altan, T., Adaptive FEM Simulation for Prediction of Variable Blank Holder Force in Conical Cup Drawing, International Journal of Machine Tools and Manufacture, Vol. 44, No. 5, (2004), pp. 487-494.

4. Wang, W.R., Chen, GL., Lin, Z.Q., and Li, S.H., Determination of Optimal Blank Holder Force Trajectories for Segmented Binders of Step Rectangle Box using PID Closed-loop FEM Simulation, International Journal of Advanced Manufacturing Technology, Vol. 32, No. 11, (2007), pp. 1074-1082.

5. Lin, Z.Q., Wang, W.R., Chen, GL., A New Strategy to Optimize Variable Blank Holder Force towards Improving the Forming Limit of Aluminum Sheet Metal Forming. Journal of Materials Processing Technology, Vol. 183, No. 2-3, (2007), pp. 339-346.

6. Kitayama, S., Hamano, S., Yamazaki, K., Kubo, T., Nishikawa H, and Kinoshita H., A Closed-loop Type Algorithm for Determination of Variable Blank Holder Force Trajectory and its Application to Square Cup Deep Drawing, International Journal of Advanced Manufacturing Technology, Vol. 51, No. 5, (2010), pp. 507-517.

7. Jansson, T., Anderson, A., and Nilson, L., Optimization of Draw-in for An Automotive Sheet Metal Part An Evaluation using Surrogate Models and Response Surfaces, Journal of Materials Processing Technology, Vol. 159, No. 3, pp. $426-434$.

8. Jakumeit, J., Herdy, M., and Nitsche, M., Parameter Optimization of the Sheet Metal Forming Process Using an Iterative Parallel Kriging Algorithm, Structural and Multidisciplinary Optimization, Vol. 29, (2005), pp. 498-507.

9. Naceur, H., Ben-Elechi, S., Batoz, J.L., and Knopf-Lenoir, C., Response Surface Methodology for The Rapid Design of Aluminum Sheet Metal Forming Parameters, Material and Design, Vol. 29, No. 4, (2008), pp. 781-790.

10. Wang, H., Enying, L., and, Li, GY., Optimization of Drawbead Design in Sheet Metal Forming Based on Intelligent Sampling by Using Response Surface Methodology, Journal of Materials Processing Technology, Vol. 206, No. 1-3, (2008), pp. 45-55.

11. Wang, H., Li, G.Y., and Zhong, Z.H., Optimization of Sheet Metal Forming Processes by Adaptive Response Surface Based on Intelligent Sampling Method, Journal of Materials Processing Technology, Vol.197, No. 1-3, (2008), pp. 77-88.

12. Wang, H., Enying, L., and Li, GY., Zhong, ZH., Optimization of Sheet Metal Forming Processes by the Use of Space Mapping Based Metamodeling Method, International Journal of Advanced Manufacturing Technology, Vol. 39, No. 7, (2008), pp. 642-655.

13. Kitayama, S., Kita, K., and Yamazaki, K., Optimization of Variable Blank Holder Force Trajectory by Sequential Approximate Optimization with RBF network, Journal of Advanced Manufacturing Technology, DOI 10.1007/s00170-011-3755-y, (Online available).

14. Bonte, M., Fourment, L., Do, T., van den Boogaard, A., and Huétink, J., Optimization of forging processes using Finite Element simulations, Structural and Multidisciplinary Optimization, Vol. 52, No. 5, (2010), pp. 797-810.

15. Kitayama, S., Arakawa, M., and Yamazaki, K., Sequential Approximate Optimization using Radial Basis Function network for engineering optimization, Optimization and Engineering, Vol. 12, No. 4, (2010), pp. 535-557.

16. Yagami, T., Manabe, K., and Yamauchi, Y., Effect of Alternating Blank Holder Motion of Drawing and Wrinkling Elimination on Deep-Drawability, Journal of Materials Processing Technology, Vol. 187-188, (2007), pp. 187-191.

17. Yagami, T., and Manabe, K., FE Analysis on Deformation Mechanism of Strain-Rate-Sensitive Materials in Cylindrical Deep-Drawing with Combination Punch Speed and Blank Holder Control, Journal of Solid Mechanics and Materials Engineering,Vol. 1, No. 12, (2007), pp. 1385-1396.

18. Ingarao, G, and Di Lorenzo, R., Optimization Methods for Complex Sheet Metal Stamping Computer Aided Engineering, Structural and Multidisciplinary Optimization, Vol. 42, (2010), pp. 459-480.

19. Hillmann, M., and Kubli, W., Optimization of Sheet Metal Forming Processes Using Simulation Programs, In: Numisheet 99 , Vol.1, (1999), pp. 287-292.

20. Guangyong, S., Guangyao, L., Shiwei, Z., Wei, X., Xuying, Y., and Qing, L., Multi-fidelity Optimization for Sheet Metal Forming Process, Structural and Multidisciplinary Optimization, Vol. 44, No. 1, (2011), pp. 111-124.

21. Miettinen, K.M., Nonlinear Multiobjective Optimization, Kluwer Academic Publisher, (1998). 